\title{
A simulation based approach for analysing benefits of workflow system integration in customer order processing
}

G. Zülch, O. Strate

ifab-Institute of Human and Industrial Engineering, University of Karlsruhe, Kaiserstrasse 12, D-76128 Karlsruhe, Tel: $++49-721-6084250$, Fax: ++49-721-69 45 57, e-mail: gert.zuelch@mach.uni-karlsruhe.de

\begin{abstract}
This paper addresses the benefit analysis for the integration of workflow management tools. A special focus is set on non-productive tasks, such as service and administration. An example is described that uses an evaluation of a process chain in customer order processing of an aluminium factory with two different levels of detail. An overall examination is done first, in order to identify possible points of improvement in the process chain. Then, a detailed analysis of these subtasks is performed. The two examinations are performed with different simulation tools by two research teams. Their findings are combined in a proof scenario to show the expected performance of the final order processing system.

Finally, results show that one crucial factor in the regarded department is the assignment of human resources to activities. Another very important aspect are the reduced processing times due to the workflow management system and advanced information technology support.
\end{abstract}

Keywords

workflow management, simulation analysis, service management 


\section{INTRODUCTION}

Many times it is difficult and cumbersome to analyse non-productive tasks in industry because they are not as precisely planned as manufacturing and assembly tasks. This is mainly because of a difficulty in setting and measuring standard times for such activities. The time duration for processing a customer request for a specific item or service may vary significantly according to the customer's ideas and desires. The customer has to interact with salespersons and office workers in order to explain his or her special requirements.

This uncertainty is a common property of service systems with high customisation. A key element of an analysis of non-productive systems is therefore the definition of processing times. This can be achieved by using existing output data of the system. In this case, the assumption is being made that an existing system, that has been in operation for a sufficiently long time, is not in a transient state anymore, so that processing times can be computed from the output data, i.e. number of completed orders, and the available capacity of human resources.

This paper describes the customer order processing of an aluminium plant. The sections of the overall production system that are modelled here encompass the customer offer creation, the shipping preparation, invoicing and the administration of claims and complaints. Production itself, being a productive task, is not modelled. There are four different products sold by the company, and the number of orders and processing times vary accordingly. In order to identify any possible benefits, two different scenarios have been simulated: the order processing chain before and after workflow re-engineering. In this context, the two scenarios are dubbed 'before' and 'after', respectively. Part of the findings were validated using an intermediate scenario.

The majority of the work was performed in a joint European project about business process re-engineering.

\section{EVALUATION}

Concerning the simulation the actual customer order processing chain - or, more precisely, a chain-like structure with only two parallel branches - is modelled using the simulation tool FEMOS. This tool is a development of the ifab-Institute of Human and Industrial Engineering at the University of Karlsruhe. It is a parameter driven simulator originally used for evaluating personnel intensive manufacturing and assembly tasks. 


\subsection{COMPARISON OF TASK ASSIGNMENT}

A major factor in simulation modelling with FEMOS are the extended capabilities as far as human qualification and ability are concerned. It is possible to assign one or more tasks to a specific worker while at the same time access to workplaces and equipment is narrowly defined. An illustration of these assignments is given in figure 1. Ability is the assignment of one operation to an employee. Feasibility denotes the assignment of an operation to a workplace. Also, the employee has to have access and knowledge about the workplace, which is called competence. If one of the three assignments is missing or is not correct, the operation can not be performed (cf. Zülch, Grobel 1992).

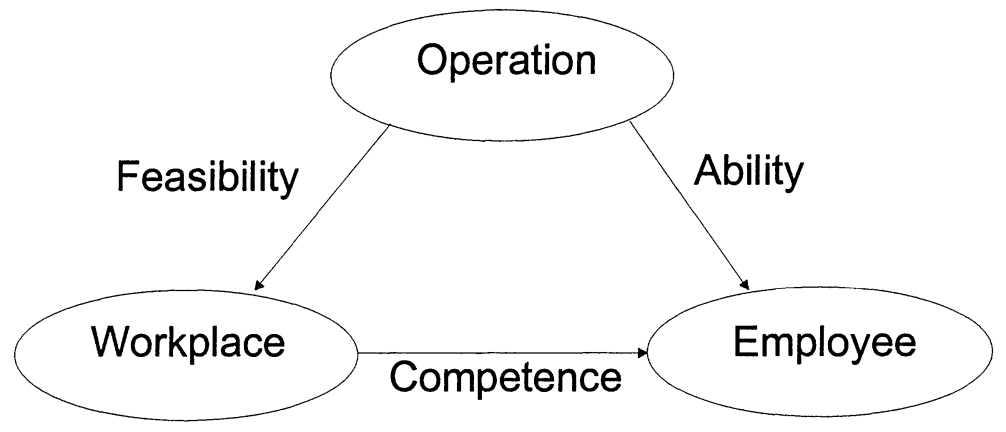

Figure 1: $\quad$ FEMOS assignment triangle

This feature is used to examine the benefit of different task assignment in the customer offer creating sub-process. In contrast to the simulation of manufacturing operations, simulation of administrative tasks and services does not always feature physical products. However, availability of information and timely decision making is crucial. When using a simulator designed for manufacturing and assembly simulation like FEMOS, inbound and outbound information is defined as material for each operation as shown in figure 2. This makes it possible to simulate unavailability or tardiness of information and decision making. If a piece of information or a decision is not made available on time, the operation can not be completed but has to wait until such piece or decision is available. For each operation, defined inbound and outbound information exists. Outbound information of one operation may be inbound information of another operation (cf. Doumeingts et al. 1999 p. 92). Consequently, related effects of information availability problems are mirrored in the system's performance.

\subsection{SIMULATION STUDY}

Historical data about the completed number of customer orders were available for processing time computation and to analyse the order arrival pattern. This pattern 
proved to be uniformly distributed. For the ease of interfacing with the simulation system FEMOS, customer orders were generated using a random number generator set to a uniform distribution. The four products strips, foil, various products, and sheets make up 38,27,18, and 17 percent of the production program, respectively. According to the company this distribution is sufficiently stable, so that the generated order spectrum features this distribution for the 'before' and 'after' scenarios.

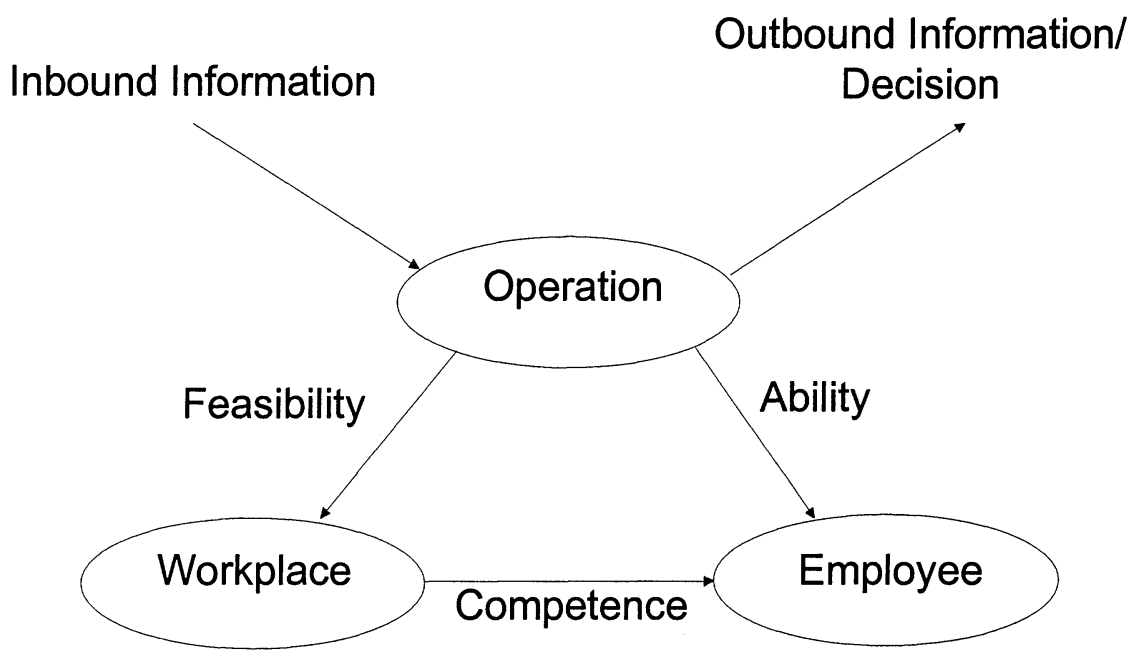

Figure 2: $\quad$ FEMOS triangle with inbound and outbound information

These data were then fed into the simulation system to simulate a 5 week period, of which the first week was discarded as the system's warm-up phase in the statistical analysis. Performance indicators of interest were of the logistic kind, namely lead time per order and number of orders finished.

$\mathrm{Aa}$ an intermediate step, a comparison of the two customer order processing systems, one with strict functional task division the other with a groupwork-like structure, was performed. Apart from these personnel assignment both systems are identical and are fed with the same input data as far as customer order arrival is concerned. It was shown that simply by changing the personnel assignment an improvement of performance can be achieved.

Since it was found that improvements are indeed possible, an in-depth analysis of the sub-process customer offer creation, depicted by operations 1 through 4 in figure 3 , was performed using a different simulation system, ARENA. This simulation system is based on a simulation modelling language called SIMAN. The advantage of this system is the ease with which decision making and branching according to various decision criteria can be modelled. It was necessary to use a different simulator since FEMOS can model information and decision availability but is limited in its modelling functionality as far as decision making and 
branching is concerned. This sub-analysis was performed by a team of researchers from the National Technical University of Athens in the same European project. Once the results of this external study were available, they were incorporated into the simulation model (cf. Doumeingts 1999 p. 131).

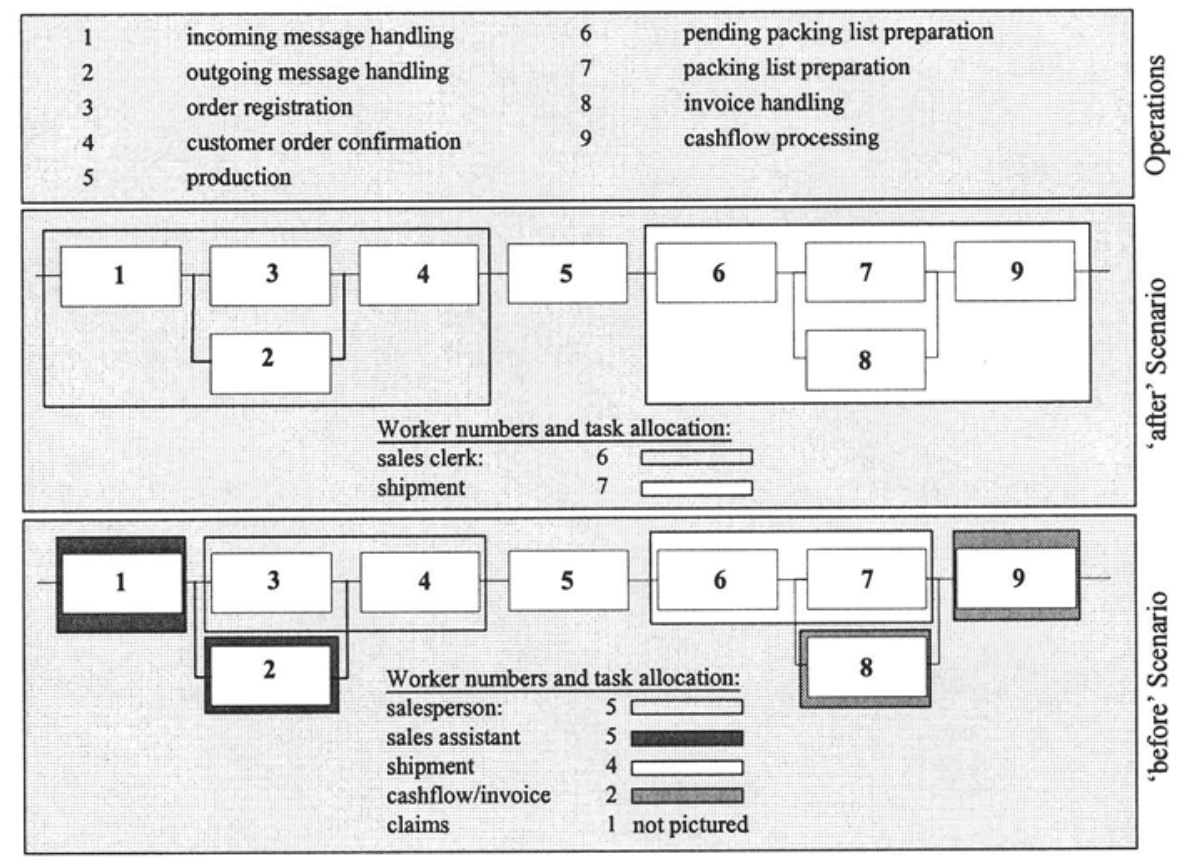

Figure 3: $\quad$ 'Before' and 'After' personnel assignments

\section{RESULTS}

The changes in personnel assignments lead to a reduction in lead times on one hand, but also to an increased effectiveness of the system. Effectiveness in this respect is meant in terms of the system's ability to cope with a higher demand of orders than initially planned. Characteristic performance is shown in figure 4. Implementation of a workflow management system and further refinements of the customer offer sub-system made it possible to reduce processing times by 40 percent. Accordingly, the number of salespersons was also reduced by 40 percent. In the 'after' system that featured all improvements and was again modelled in FEMOS, this solution proved to be as effective as the solution in the intermediate scenario with the original number of salespersons and the changed assignments. It can therefore be concluded that a reduction of the sales workforce by 40 percent is 
feasible (Doumeingts et al. 1999 p.149). Results are depicted in figure 4 below for the average lead time.
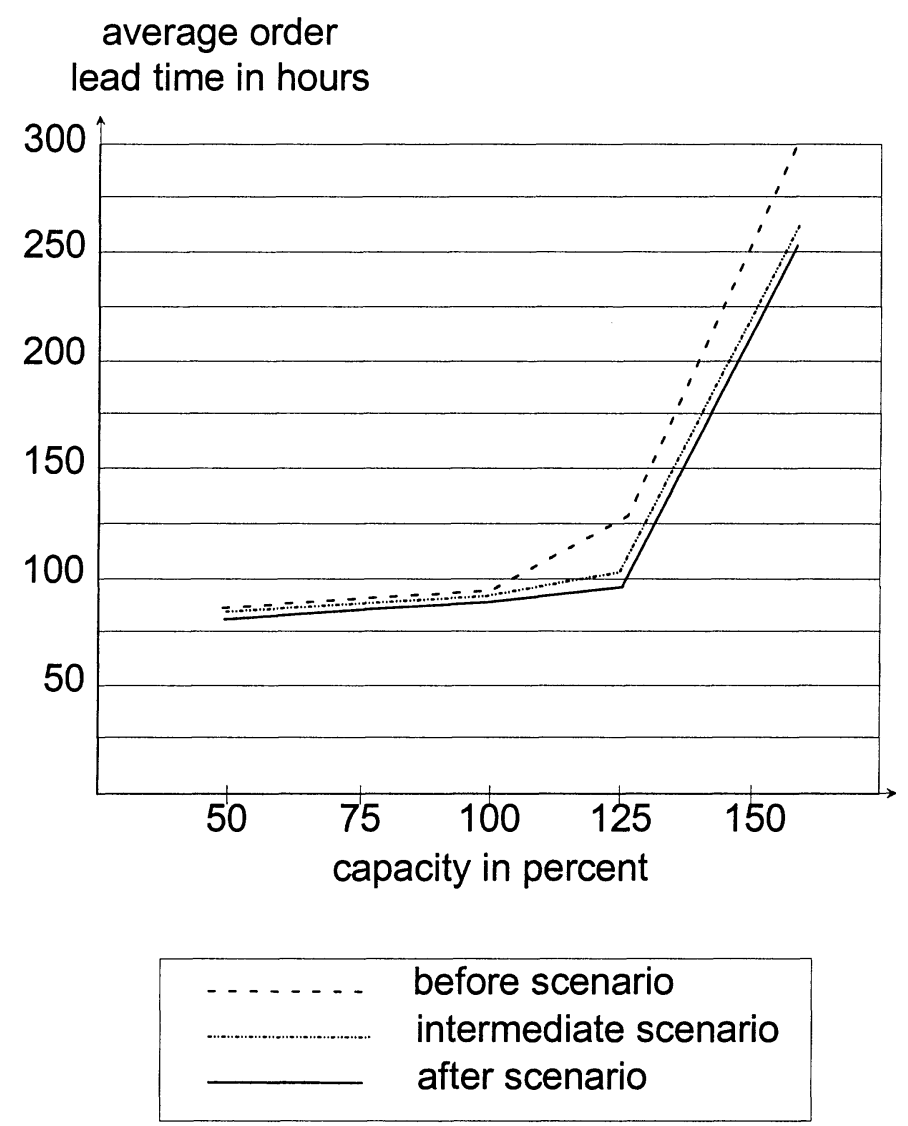

Figure 4: $\quad$ Lead time simulation results

A major drawback is that the effect of the workflow system in terms of processing time reduction can only be estimated in the simulation. In reality, the brevity with which the system has been used in the company does not permit to make too reliable a conclusion. To ease this problem and to find a possible target performance for improvement, a sensitivity analysis on the capacity requirement was performed. The capacity requirement is defined as the processing time multiplied with the number of orders for a given product. With this additional information it can easily be seen, how the change in order numbers, the change in processing times (e.g. due to moving down on the learning curve for using the new workflow management system), or both impact the performance of the overall system. 
The system's performance shows that lead time does decrease due to a change in assignment, but it remains at a high level. This is caused by many customer orders being delayed in distribution and shipping. Based on this conclusive study of the customer order processing chain and the benefits gained in the offer creation process in particular, it is recommended to conduct an in-depth study of the distribution and shipping process as well. The results of this additional study could be a further reduction in overall lead time due to improved distribution and shipping, extending the benefits of the installed information technology for the user.

\section{BIBLIOGRAPHY}

Doumeingts, Guy; et. al. (1999) A Methodology for Re-engineering and Information Technology Implementation. Shaker Publishing, Aachen.

Zülch, Gert and Grobel, Thomas (1992) Simulating the Departmental Organization for Production to Order. In: One-of-a-kind Production: New approaches (Edt. Hirsch, B; Thoben, K.-D.). North Holland Publisher, Amsterdam et al.

\section{BIOGRAPHY}

Gert Zülch is full professor at the University of Karlsruhe and head of the Institute for Human and Industrial Engineering. He has been active in national and international research activities in the field of ergonomics, industrial engineering and industrial management for more than 13 years.

Oliver Strate is researcher at the University of Karlsruhe. His activities include simulation studies, organisation design and analysis, as well as production planning and control. His work encompasses manufacturing and service systems. 